\title{
Dark-matter electric and magnetic dipole moments
}

\author{
Kris Sigurdson, ${ }^{1, *}$ Michael Doran, ${ }^{2}$ Andriy Kurylov, ${ }^{1}$ Robert R. Caldwell, ${ }^{2}$ and Marc Kamionkowski ${ }^{1}$ \\ ${ }^{1}$ California Institute of Technology, Mail Code 130-33, Pasadena, California 91125, USA \\ ${ }^{2}$ Department of Physics and Astronomy, Dartmouth College, 6127 Wilder Laboratory, Hanover, New Hampshire 03755, USA
}

(Received 17 June 2004; published 6 October 2004)

\begin{abstract}
We consider the consequences of a neutral dark-matter particle with a nonzero electric and/or magnetic dipole moment. Theoretical constraints, as well as constraints from direct searches, precision tests of the standard-model, the cosmic microwave background and matter power spectra, and cosmic gamma rays, are included. We find that a relatively light particle with mass between an $\mathrm{MeV}$ and a few $\mathrm{GeV}$ and an electric or magnetic dipole as large as $\sim 3 \times 10^{-16} e \mathrm{~cm}$ (roughly $1.6 \times 10^{-5} \mu_{B}$ ) satisfies all experimental and observational bounds. Some of the remaining parameter space may be probed with forthcoming more sensitive direct searches and with the Gamma-Ray Large Area Space Telescope.
\end{abstract}

DOI: 10.1103/PhysRevD.70.083501

\section{INTRODUCTION}

A wealth of observational evidence indicates the existence of considerably more mass in galaxies and clusters of galaxies than we see in stars and gas. The source of the missing mass has been a problem since Zwicky's 1933 measurement of the masses of extragalactic systems [1]. Given the evidence from galaxy clusters, galaxy dynamics and structure formation, big-bang nucleosynthesis, and the cosmic microwave background that baryons can only account for $\sim 1 / 6$ of this matter, most of it must be nonbaryonic. Although neutrinos provide the cosmological density of dark-matter if their masses sum to $\sim 12 \mathrm{eV}$, such particles cannot (essentially from the Pauli principle) have a sufficiently high phase-space density to account for galactic dark-matter halos [2]; moreover, such masses are now inconsistent with neutrino-mass measurements [3]. Theorists have thus taken to considering for dark-matter candidates new physics beyond the standard-model. To date, the most promising candidates - those that appear in fairly minimal extensions of the standard-model and which coincidentally have a cosmological density near the critical density-are a weakly-interacting massive particle (WIMP), such as the neutralino, the supersymmetric partner of the photon, $Z^{0}$ boson, and/or Higgs boson [4], or the axion [5]. A considerable theoretical literature on the properties and phenomenology of these particles has arisen, and there are considerable ongoing experimental efforts to detect these particles.

In the absence of discovery of such particles, it may be well worth exploring other possibilities. Thus, an alternative line of investigation takes a more modelindependent approach and seeks to explore phenomenologically the possible properties of a dark-matter particle. Along these lines, for example, constraints to stronglyinteracting dark-matter were considered in Ref. [6]; selfinteracting dark-matter has been considered $[7,8]$, and

*Electronic address: ksigurds@tapir.caltech.edu
PACS numbers: 95.35.+d, 13.40.Em, 14.80.-j, 95.30.Cq

some have studied whether dark-matter might be charged [9] or have a millicharge $[10,11]$.

Our investigation follows in spirit the latter possibility. In particular, dark-matter is so called because the coupling to photons is assumed to be nonexistent or very weak, or else we would have presumably seen such particles either through absorption or emission of radiation or in laboratory experiments. In this paper, we ask the question, 'How dark is 'dark'?" In other words, how weak must the coupling of the dark-matter particle to the photon be in order to be consistent with laboratory and astrophysical constraints? In the work on millicharged particles, a dark-matter coupling to photons was assumed to arise from a tiny charge.

In this paper we consider the possibility that the darkmatter possesses an electric or magnetic dipole moment. The punch line, illustrated in Fig. 1, is that a Dirac particle with an electric or magnetic dipole moment of order $\sim 10^{-17} e \mathrm{~cm}$ with a mass between an MeVand a few $\mathrm{GeV}$ can provide the dark-matter while satisfying all experimental and observational constraints. ${ }^{1}$

In the following Section, we introduce the effective Lagrangian for the dipolar-dark-matter (DDM) interaction with photons. We discuss the relic abundance in Section III. Section IV presents constraints on darkmatter dipole moments and masses that arise from direct searches at low-background detectors as well as constraints from high-altitude experiments. Section V discusses constraints due to precision tests of the standardmodel, while Section VI discusses constraints due to the cosmic microwave background and the growth of largescale structure. We provide some concluding remarks in

\footnotetext{
${ }^{1}$ Throughout, we will quote numbers for both the electric and magnetic dipole moments in units of $e \mathrm{~cm}$, where $e$ is the electron charge. For reference, the Bohr magneton $\mu_{B}=$ $e \hbar / 2 m_{e}=1.93 \times 10^{-11} e \mathrm{~cm}$ in these units. Also note that we work in rationalized Gaussian units so that the fine-structure constant $\alpha \equiv e^{2} / 4 \pi \hbar c \approx 1 / 137$, and in particle-physics units $(\hbar=c=1) e^{2} \approx 4 \pi / 137$ and $e \approx 0.303$.
} 


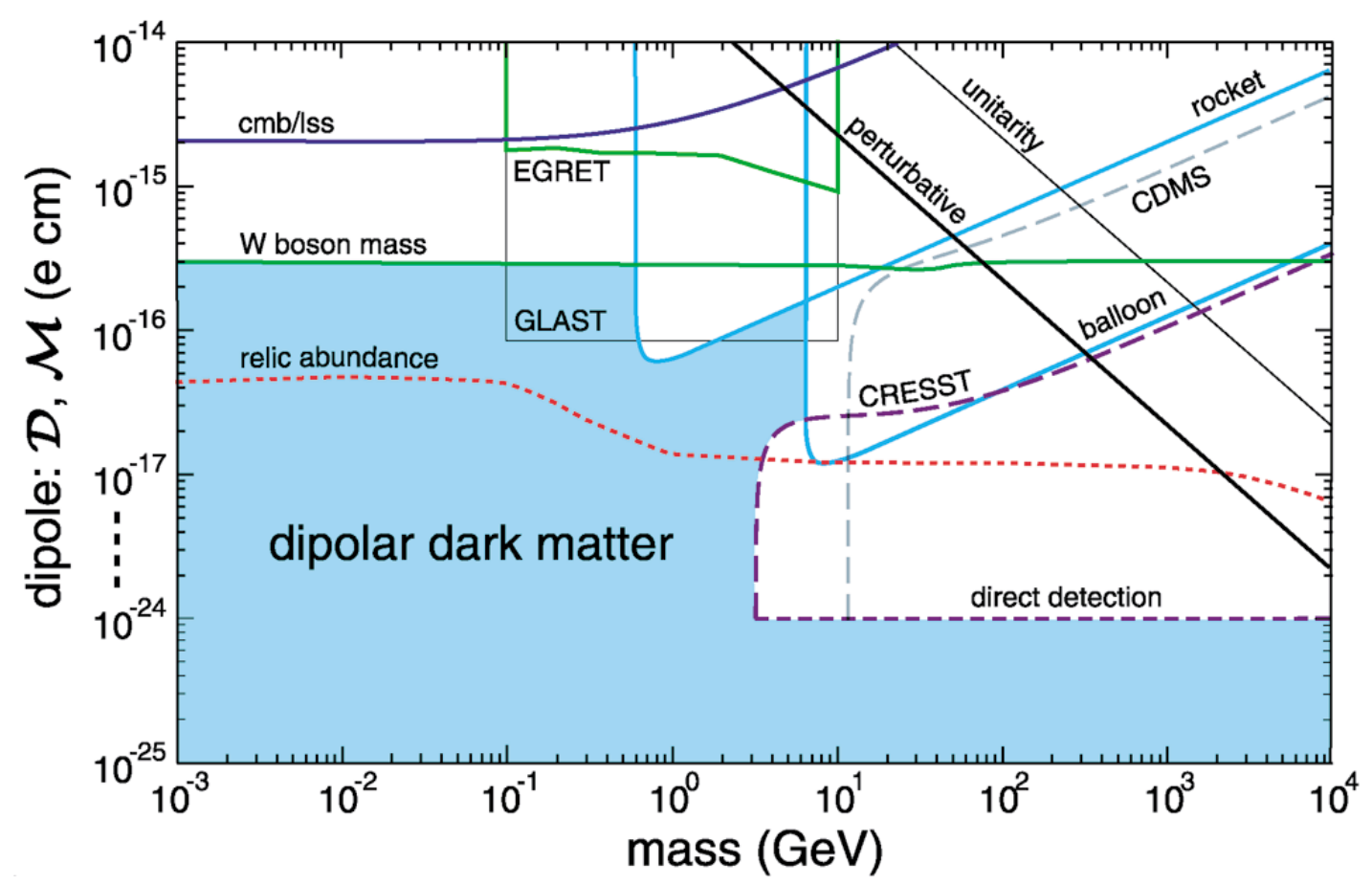

FIG. 1 (color online). The constraints on the dipolar-dark-matter parameter space $\left[m_{\chi},(\mathcal{D}, \mathcal{M})\right]$ that come from present day searches and experiments. Viable candidates must lie in the shaded region, below the solid lines and outside the long-dashed lines. The short-dashed "relic abundance" curve shows where the dark-matter would have a cosmological density $\Omega_{\chi} h^{2}=0.135$, assuming standard freeze-out, no particle-antiparticle asymmetry, and no interactions with standard-model particles apart from the dipole coupling to photons. Note that the EGRET and GLAST curves constrain the combination $\left(\mathcal{D}^{4}+\mathcal{M}^{4}\right)^{1 / 4}$, the perturbative and unitarity curves apply to the stronger of $(\mathcal{D}, \mathcal{M})$, while all other curves restrict $\left(\mathcal{D}^{2}+\mathcal{M}^{2}\right)^{1 / 2}$.

Section VII. The Appendix provides details of the calculation of the drag-force between the baryon and DDM fluids used in Section VI.

\section{THEORY OF DIPOLE MOMENTS}

A particle with a permanent electric and/or magnetic dipole moment must have a nonzero spin; we thus consider spin-1/2 particles. Moreover, Majorana particles cannot have permanent dipole moments, so we consider Dirac fermions. Since the spin and the magnetic dipole are both axial vectors, a magnetic dipole moment can arise without violating any discrete symmetries. However, the electric dipole moment is a vector and thus requires time-reversal and parity violation.

The effective Lagrangian for coupling of a Dirac fermion $\chi$ with a magnetic dipole moment $\mathcal{M}$ and an electric dipole moment $\mathcal{D}$ to the electromagnetic field $F^{\mu \nu}$ is

$$
\mathcal{L}_{\gamma \chi}=-\frac{i}{2} \bar{\chi} \sigma_{\mu \nu}\left(\mathcal{M}+\gamma_{5} \mathcal{D}\right) \chi F^{\mu \nu}
$$

At energies low compared to the dark-matter mass, the photon is blind to distinctions between $\mathcal{M}$ and $\mathcal{D}$ (unless time-reversal-violating observables are considered). Hence, we can discuss limits to $\mathcal{D}$ which equally apply to $\mathcal{M}$, except where noted.
On dimensional grounds, we expect the electric dipole moment to satisfy the limit $\mathcal{D} \lesssim e m_{\chi}^{-1} \simeq 2 \times$ $10^{-14}\left(m_{p} / m_{\chi}\right) e \mathrm{~cm}$, where $m_{p}$ is the proton mass. Similar arguments also apply to the magnetic dipole moment. ${ }^{2}$ This limit is shown as the perturbative bound in Fig. 1, as violation of this bound would signal some nontrivial or nonperturbative field configuration. As we will see below, more rigorous but slightly weaker upper limits can be set with unitarity arguments.

These upper limits already simplify our analysis. The phenomenology of charged dark-matter particles is determined largely by the ability of these particles to form atom-like bound states with electrons, protons, or each other. However, dipolar-dark-matter cannot form such bound states. A neutral particle with a magnetic-moment will not form bound states with charged particles. Curiously enough, a neutral particle with an electric dipole moment (EDM) can indeed form a bound state with an electron, as first noted by no less than Fermi and Teller [12], but only if the dipole moment is greater than $0.639 e a_{0}=3.4 \times 10^{-9} e \mathrm{~cm}$ (assuming $m_{\chi} \gg m_{e}$ ) where $a_{0}$ is the Bohr radius. For smaller values of the dipole, the

\footnotetext{
${ }^{2}$ The limit is satisfied if $m_{\chi}$ is the lightest scale relevant for the DDM sector (see Section V for discussion). Note, however, that the actual magnitude of the dipole moments in a particular theory can be significantly below this limit.
} 
electron "sees" both poles of the dipole and finds no stable orbit. This critical electric dipole moment scales inversely with the dipole-electron reduced mass, so a bound state with a proton can occur if the dipole mass is $\gg m_{p}$ and $\mathcal{D} \gtrsim 1.8 \times 10^{-12} e \mathrm{~cm}$. As we will see below, such values for the EDM cannot occur for a pointlike DDM. Likewise, the weakness of the dipole-dipole interaction prevents the formation of any stable darkmatter atoms.

The first cosmological constraint is that from big-bang nucleosynthesis (BBN). BBN requires that the effective number of relativistic degrees of freedom at $T \sim \mathrm{MeV}$ does not exceed the equivalent of roughly 0.2 neutrino species [13]. Since the particles we are considering are Dirac particles, they act like two effective neutrino species and thus cannot be relativistic and in equilibrium at BBN. Generally, such considerations rule out $m_{\chi} \lesssim \mathrm{MeV}$, and so we restrict our attention in Fig. 1 to masses above an $\mathrm{MeV}$. Strictly speaking, if the dipole moment is $(\mathcal{D}, \mathcal{M}) \lesssim 10^{-22} e \mathrm{~cm}$, and if the particle has no other interactions with standard-model particles, then a particle of mass $\lesssim \mathrm{MeV}$ can decouple at a temperature $\gtrsim$ $10 \mathrm{GeV}$, and if so, it will evade the BBN limit.

\section{DARK-MATTER ANNIHILATION AND RELIC ABUNDANCE}

DDM particles can exist in thermal equilibrium in the early Universe when the temperature $T \gg m_{\chi}$, and their interactions will freeze out when $T$ drops below $m_{\chi}$ resulting in some cosmological relic abundance. The mass density of relic DDM particles is fixed by the cross-section $\sigma_{\text {ann }}$ for annihilation to all lighter particles times the relative velocity $v$ through (see, e.g., Eq. (5.47) in Ref. [14]),

$$
\begin{aligned}
\Omega_{\chi} h^{2} \simeq & 3.8 \times 10^{7}\left(\frac{m_{\chi}}{m_{p}}\right) \ln (A / \sqrt{\ln A}) / A \\
= & 0.135\left(g_{*} / 10\right)^{-1 / 2}\left(\frac{\sigma_{\mathrm{ann}} v}{5.3 \times 10^{-26} \mathrm{~cm}^{3} \mathrm{sec}^{-1}}\right)^{-1} \\
& \times \frac{\ln [A / \sqrt{\ln A}]}{21},
\end{aligned}
$$

where

$$
\begin{aligned}
A & =0.038 \sqrt{g_{*}} m_{p l} m_{\chi}\left(\sigma_{\mathrm{ann}} v\right) \\
& =\frac{6.3 \times 10^{9}\left(g_{*} / 10\right)^{1 / 2}}{\left(m_{\chi} / \mathrm{GeV}\right)}\left(\frac{\sigma_{\mathrm{ann}} v}{5.3 \times 10^{-26} \mathrm{~cm}^{3} \mathrm{sec}^{-1}}\right)
\end{aligned}
$$

assuming that annihilation takes place (as it does in our case) through the $s$ wave. Here, $g_{*}$ is the effective number of relativistic degrees of freedom at the temperature $T_{f} \sim$ $m_{\chi} / A$ of freeze-out. For the interaction of Eq. (1), DDM-anti-DDM pairs can annihilate to either two photons or to charged particle-antiparticle pairs through the diagrams shown in Figs. 2 and 3. The cross sections for these two processes (to lowest-order in $v$ ) are

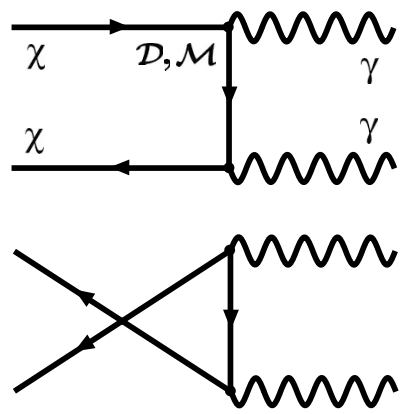

FIG. 2. Feynman diagrams for annihilation of a DDM-antiDDM pair to two photons.

$$
\begin{aligned}
\sigma_{\chi \bar{\chi} \rightarrow 2 \gamma} v & =\left(\mathcal{D}^{4}+\mathcal{M}^{4}\right) m_{\chi}^{2} / 2 \pi \\
& =1.0 \times 10^{-33} m_{\mathrm{GeV}}^{2}\left(\mathcal{D}_{17}^{4}+\mathcal{M}_{17}^{4}\right) \mathrm{cm}^{3} \mathrm{sec}^{-1}, \\
\sigma_{\chi \bar{\chi} \rightarrow f \bar{f}} v & =N_{\mathrm{eff}} \alpha\left(\mathcal{D}^{2}+\mathcal{M}^{2}\right) \\
& =2 \times 10^{-27} N_{\mathrm{eff}}\left(\mathcal{D}_{17}^{2}+\mathcal{M}_{17}^{2}\right) \mathrm{cm}^{3} \mathrm{sec}^{-1},
\end{aligned}
$$

where $\left(\mathcal{D}_{17}, \mathcal{M}_{17}\right)=(\mathcal{D}, \mathcal{M}) /\left(10^{-17} e \mathrm{~cm}\right)$, and $m_{\mathrm{GeV}} \equiv$ $m_{\chi} / \mathrm{GeV}$. Here, $N_{\text {eff }}=\sum_{f} Q_{f}^{2} N_{c f}$ is the effective number of fermion-antifermion pairs with mass $m_{f}<m_{\chi}, Q_{f}$ is the charge of fermion $f$, and $N_{c f}$ is the number of color degrees of freedom for fermion $f$. $\left(N_{c f}=1\right.$ for electrons.) In the standard-model, annihilation can also occur to $W^{+} W^{-}$pairs above threshold. For $\left(\mathcal{D}_{17}, \mathcal{M}_{17}\right) \times$ $\left(m_{\chi} / m_{p}\right) \lesssim 5000$, fermions are the dominant final-state. The present day mass density of DDM particles thus depends primarily on the dipole moment. If such particles are to account for the dark-matter, then $\Omega_{\chi} h^{2}=0.135$ [15], and $\left(\mathcal{D}^{2}+\mathcal{M}^{2}\right)^{1 / 2} \simeq 1.0 \times 10^{-17} e \mathrm{~cm}$ for $m_{\chi} \sim$ $1 \mathrm{GeV}$. The full mass dependence of this result is shown in Fig. 1.

The cross sections in Eq. (4) are $s$-wave cross sections. According to partial-wave unitarity, the total $s$-wave annihilation cross-section must be $\sigma \lesssim 4 \pi / m_{\chi}^{2}$ [16], so that $(\mathcal{D}, \mathcal{M}) m_{\chi} \lesssim 3$, fixed by the cross-section for annihilation to two photons. This limit is shown in Fig. 1, as is the more stringent, but less rigorous, limit $(\mathcal{D}, \mathcal{M}) \lesssim$ $e / m_{\chi}$.

Of course, the present day mass density of DDM particles could differ from the estimates obtained above. To

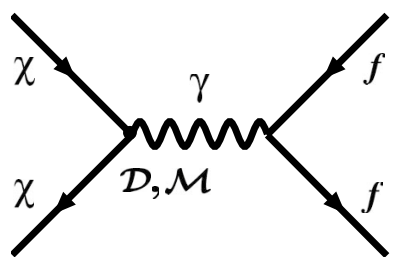

FIG. 3. Feynman diagram for DDM-anti-DDM annihilation to fermion-antifermion pairs. 
obtain these results, we assumed (i) that the dipole interaction with photons is the only interaction of these particles; and (ii) that there is no particle-antiparticle asymmetry. It is reasonable to assume that in any realistic model, a dark-matter dipole interaction will arise from loop diagrams involving other standard-model and new particles. If so, then there may be other contributions to the annihilation cross sections. In this case, the relic abundance will be smaller than we have estimated above. We thus conclude that if there is no particle-antiparticle asymmetry, our estimates should be treated as an upper bound to the relic abundance, and the $\Omega_{\chi} h^{2}$ curve in Fig. 1 should thus be considered an upper limit to the desired values of $\mathcal{D}$ and $\mathcal{M}$. On the other hand, the relic abundance could also be increased if exotic processes increase the expansion rate during freeze-out [17].

If there is an asymmetry between $\chi$ and $\bar{\chi}$, then the relic abundance is increased relative to our estimate. In this case, however, the present day Universe should contain predominantly either particles or antiparticles. Although there is no a priori reason to expect there to be a particle-antiparticle asymmetry, the observed baryon-antibaryon asymmetry might lead us to expect an analogous dark-matter asymmetry, should the darkmatter be composed of Dirac particles. It is possible such asymmetries have a common origin.

Finally, we have assumed above that the particles freeze out when they are nonrelativistic. However, as the dipole moment is lowered for a given mass, freeze-out occurs earlier. If the dipole moment is reduced beyond a certain value, and if there are no other couplings to standard-model particles, then freeze-out will be relativistic. These particles will then be roughly as abundant as photons, and they will overclose the Universe by huge margins unless their masses are $\lesssim$ few eV; even in this case they will violate constraints to hot dark-matter from the CMB and large-scale structure, and they will also be unable, from the Tremaine-Gunn argument, to make up the dark-matter in Galactic halos. The transition from nonrelativistic to relativistic freeze-out occurs (again, assuming no nondipole interactions with standard-model particles) for $m_{\chi} \mathcal{D}_{17}^{2} \lesssim 10^{-10} \mathrm{GeV}$ for $m_{\chi} \gtrsim \mathrm{MeV}$, and for $m_{\chi} \lesssim \mathrm{MeV}$, at $m_{\chi} \mathcal{D}_{17}^{4 / 3} \lesssim 200 \mathrm{MeV}$.

\section{DIRECT-DETECTION}

The diagram for scattering of a DDM-particle with a particle of charge $\mathrm{Ze}$ occurs through the exchange of a photon, as shown in Fig. 4 (not unlike the electronneutron interaction [18]). In the nonrelativistic limit, the differential cross-section for this process is given by,

$$
\frac{d \sigma}{d \Omega}=\frac{Z^{2} e^{2}\left(\mathcal{D}^{2}+\mathcal{M}^{2}\right)}{8 \pi^{2} v^{2}(1-\cos \theta)}
$$

where $v$ is the relative velocity. Through this interaction

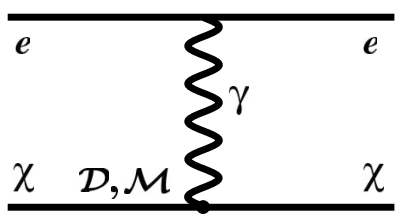

FIG. 4. Feynman diagram for elastic-scattering of an electron from a DDM-particle.

the DDM might produce a signal in a direct-detection experiment. Although the total cross-section is formally infinite, the divergence comes from the small-momentum-transfer scatterings that will be screened by atomic electrons. Roughly speaking, then, the DDM-nucleus elastic-scattering cross-section will be $\sigma \sim(Z e)^{2}\left(\mathcal{D}^{2}+\right.$ $\left.\mathcal{M}^{2}\right) / 2 \pi v^{2} \simeq 6.4 \times 10^{-32} Z^{2}\left(\mathcal{D}_{17}^{2}+\mathcal{M}_{17}^{2}\right) \mathrm{cm}^{2}$, using $v \sim 10^{-3} c$. Current null searches in germanium detectors $[(A, Z)=(76,32)]$ correspond for masses $m_{\chi} \sim 10 \mathrm{GeV}$ to a rough upper limit to the cross-section $\sim 10^{-42} \mathrm{~cm}^{2}[19]$, thus ruling out any dipole moment $\left(\mathcal{D}_{17}^{2}+\mathcal{M}_{17}^{2}\right)^{1 / 2} \gtrsim 10^{-7}$. This is shown in Fig. 1 as the horizontal dashed line at $\mathcal{D}=10^{-24} e \mathrm{~cm}$. Note that the cross-section limit depends (and increases) with mass at higher masses; the curve appears as a horizontal line simply because of the break in scale on the $y$ axis.

This seems like a very stringent limit, especially considering the value, $\mathcal{D}_{17} \sim 1$, favored for the correct cosmological density. However, if the dipole moment becomes large enough, the particles will be slowed in the rock above the detector and thus evade detection in these underground experiments. In order to determine the magnitude of the dipole moment for this to occur, we need to calculate the stopping power $d E / d x$ for the particle as it passes through the atmosphere and then the rock. Since elastic-scattering takes place through exchange of a photon, it leads to a long-range interaction and, as we have seen above, a formally divergent elasticscattering cross-section. The calculation of the stopping power thus parallels that for ionization loss due to Coulomb collisions, with two important differences. First of all, since the long-range force is $\propto r^{-3}$, as opposed to $r^{-2}$ for Rutherford scattering, stopping occurs via scattering from nuclei, rather than electrons. Second, since this interaction falls more rapidly with radius than the Coulomb interaction, the stopping power is due primarily to hard scatters at small impact parameter, rather than soft scatters at a wide range of impact parameters.

Our result for the stopping power due to scattering from nuclei of charge $Z$ is

$$
\frac{d E}{d x}=-n_{N} \int T d \sigma=-n_{N} \frac{(Z e)^{2}\left(\mathcal{D}^{2}+\mathcal{M}^{2}\right) \mu^{2} c^{2}}{2 \pi m_{N}}
$$

where the kinetic-energy transfer in a single collision is $T=p^{2}(1-\cos \theta) / m_{N}, \quad x$ is the depth, and $\mu=$ $\mu\left[m_{\chi}, m_{N}\right]=m_{\chi} m_{N}\left(m_{\chi}+m_{N}\right)^{-1}$ is the reduced mass. 
For very weak WIMP interactions with nuclei, the most restrictive limits on the WIMP-proton cross-section (the smallest upper bounds) are obtained from null searches from experiments that are deepest underground (so as to reduce the background). However, the most restrictive constraints on the cross-section at the upper end of the excluded range of cross sections will come from the shallowest underground experiment with a null result. From Eq. (6), we find that the dark-matter particles will only penetrate to a depth $x=\left(E_{i}-E_{f}\right) /|d E / d x|$ where $E_{i}=\frac{1}{2} m_{\chi} v^{2}$ is the initial dark-matter kineticenergy and $E_{f}$ is the final energy. For a stopped particle, $E_{f}=0$. However, the particle only needs to lose enough energy for $E_{f}$ to drop below the detection threshold for a particular experiment. Equating the maximum kineticenergy transferable in a collision $(\theta=\pi)$ to the threshold detectable nuclear-recoil energy $\left(E_{\mathrm{th}}\right)$, we find the velocity must be slowed to $v_{f}^{2}=m_{d} E_{\mathrm{th}} / 2 \mu\left[m_{\chi}, m_{d}\right]^{2}$, where $m_{d}$ is the mass of the nuclei in the detector, and $\mu\left[m_{\chi}, m_{d}\right]$ is the DDM-nucleus reduced mass. Hence, the final darkmatter energy must be $E_{f}=m_{\chi} m_{d} E_{\mathrm{th}} / 4 \mu\left[m_{\chi}, m_{d}\right]^{2}$.

So far we have assumed that the particle loses energy but is not significantly deflected in each scatter; this will be a good approximation if $m_{\chi} \gg m_{N}$. However, when $m_{\chi} \lesssim m_{\mathrm{Si}} \simeq 26 \mathrm{GeV}$, the dark-matter particle may be backscattered upon encountering a terrestrial nucleus, rather than simply being slowed without deflection. In this case, the particle will diffuse, undergoing $N \sim$ $m_{N} / 2 m_{\chi}$ scatters before coming to rest. If so, the penetration depth will be reduced by an additional factor of $\sim N^{-1 / 2}$. We thus replace $d E / d x \rightarrow d E / d x[1+$ $\left.\left(m_{N} / 2 m_{\chi}\right)^{1 / 2}\right]$ in our expression for the penetration depth.

Then, for a given shielding thickness $L$, in meters water equivalent (mwe), we invert the expression for the stopping distance to obtain the following bound on the dipole strength:

$$
\mathcal{D}^{2}+\mathcal{M}^{2}>\frac{\frac{1}{2} m_{\chi} v^{2}-\frac{1}{4} \frac{m_{\chi} m_{d}}{\mu\left[m_{\chi}, m_{d}\right]^{2}} E_{\mathrm{th}}}{\frac{e^{2}}{2 \pi} L \sum_{i} f_{i} Z_{i}^{2} \frac{\mu\left[m_{\chi}, m_{i}\right]^{2}}{m_{i}^{2}}\left[1+\left(m_{i} / 2 m_{\chi}\right)^{1 / 2}\right]},
$$

where the index $i$ sums over the composition of the shielding material, and $f_{i}$ is the fractional composition by weight. We use a realistic model of the composition of the Earth (chemical composition by weight: O [46.6\%], Si [27.7\%], $\mathrm{Al}$ [8.1\%], $\mathrm{Fe}$ [5\%], Ca [3.6\%], Na [2.8\%], K [2.6\%], $\mathrm{Mg}$ [2.1\%]) and atmosphere (10 mwe consisting of a 4:1 ratio of nitrogen to oxygen), although the resulting bounds do not change substantially if we ignore the atmosphere and approximate the Earth's crust as entirely composed of Si. We take the initial DDM velocity to be $300 \mathrm{~km} \mathrm{sec}^{-1}$.

The shallowest underground experiment with a strong null result is the Stanford Underground Facility run of the Cryogenic Dark-Matter Search (CDMS) [20], which was situated at a depth of 16 mwe. With a detector energy threshold of $E_{\text {th }}=5 \mathrm{keV}$, it is sensitive to DDM masses down to $m_{\chi} \sim 10 \mathrm{GeV}$. Near this threshold we find that DDM particles are stopped by the shielding for $\mathcal{D}_{17} \gtrsim$ 20. This bound grows more prohibitive with increasing mass, as illustrated in Fig. 1. The Cryogenic Rare Event Search with Superconducting Thermometers (CRESST) [21], though at a depth of 3800 mwe, extends to slightly lower masses, having a detector threshold energy $E_{\mathrm{th}}=$ $0.6 \mathrm{keV}$. Near $m_{\chi} \sim 1 \mathrm{GeV}$ the minimum dipole strength is $\mathcal{D}_{17} \geq 2$. However, there are no limits from underground experiments for DDM masses below $1 \mathrm{GeV}$.

Two airborne experiments - unobscured by the atmosphere or rock-which have closed the windows on some forms of strongly-interacting dark-matter [6,22], also restrict dark-matter dipole moments. To determine the predicted signal at a detector, we recast Eq. (5) as the cross-section per energy transfer, whereby $d \sigma / d T=$ $Z^{2} e^{2}\left(\mathcal{D}^{2}+\mathcal{M}^{2}\right) / 4 \pi v^{2} T$. The event rate (per time, energy, and unit mass of detector) is

$$
\begin{aligned}
R & =N_{N}\left(0.3 \mathrm{GeV} \mathrm{cm}^{2}\right) \frac{v}{m_{\chi}} \frac{d \sigma}{d T} \\
& =2.3\left(\mathcal{D}_{17}^{2}+\mathcal{M}_{17}^{2}\right) \frac{m_{p}}{m_{\chi}}\left(\frac{\mathrm{keV}}{T}\right) \mathrm{sec}^{-1} \mathrm{keV}^{-1} \mathrm{~g}^{-1},
\end{aligned}
$$

where $N_{N}$ is the number of nuclei per gram of material.

The silicon semiconductor detector flown on a balloon in the upper atmosphere by Rich et al. [23] observed an event rate of $\sim 0.5$ counts $\mathrm{sec}^{-1} \mathrm{keV}^{-1} \mathrm{~g}^{-1}$ nuclear recoils in the lowest energy bin at $2 \mathrm{keV}$. For dark-matter masses above the threshold $\sim 7 \mathrm{GeV}$, we thus require $\left(\mathcal{D}_{17}^{2}+\mathcal{M}_{17}^{2}\right)\left(m_{p} / m_{\chi}\right)<0.2$.

The X-ray Quantum Calorimeter (XQC) detector flown on a rocket by McCammon et al. [24], was designed to probe the soft $\mathrm{x}$-ray background. However, it serendipitously provides a tight constraint on dipolar-dark-matter. To predict the expected number of events, we start by computing the number of DDM particles that could impinge on the detector: $N_{\chi}=n_{\chi} v A t=3 \times 10^{7} m_{p} / m_{\chi}$, where $n_{\chi}$ is the galactic number density of dark-matter particles, $v$ is their velocity, the cross-sectional area of the XQC detector is $A=0.33 \mathrm{~cm}^{2}$, and the rocket flight was about $t \sim 120$ seconds. The chief property of the $\mathrm{XQC}$ detector is the $14 \mu \mathrm{m}$ thick Si substrate above the thermistor, providing a target of $N_{N} \sim 6.5 \times$ $10^{19}$ nuclei $/ \mathrm{cm}^{2}$. Thus, the event/energy count $N_{N} N_{\chi} d \sigma / d T$ integrated over the 25-100 eV energy bin gives a predicted $\sim 0.38\left(\mathcal{D}_{17}^{2}+\mathcal{M}_{17}^{2}\right)\left(m_{p} / m_{\chi}\right)$ events compared to the $\sim 10$ observed events. Since the detector has a $25 \mathrm{eV}$ threshold, energy transfer by dark-matter particles as light as $\sim 1 \mathrm{GeV}$ can be detected. Altogether, the balloon and rocket experiments exclude a wide range of dipole strengths and masses, as illustrated in Fig. 1. 


\section{CONSTRAINTS FROM PRECISION MEASUREMENTS}

We now consider the limits placed on DDM due to precision tests of the standard-model. Our use of perturbation theory is valid provided the energy scale of the interaction $\mathcal{E}$ satisfies $(\mathcal{D}, \mathcal{M}) \mathcal{E} \ll 1$. In addition, we require that the DDM mass satisfies $(\mathcal{D}, \mathcal{M}) m_{\chi} \lesssim 1$, equivalent to the unitary bound [16], which ensures the self-consistency of the local operator in Eq. (1). Indeed, if $\Lambda$ is the energy scale at which a dipole is generated then one generally expects $(\mathcal{D}, \mathcal{M}) \Lambda \sim 1$. In $\mathcal{L}_{\gamma \chi}$ we assume that all interacting particles with masses greater than $\Lambda$ have been integrated out. Consequently, one must have at least $m_{\chi}<\Lambda$ for the dark-matter to be dynamical, which also yields $(\mathcal{D}, \mathcal{M}) m_{\chi} \lesssim 1$.

\section{A. Muon Anomalous Magnetic-Moment}

The interaction in Eq. (1) contributes to the photon propagator via the diagram shown in Fig. 5. The photonDDM interaction vertices are either both electric or magnetic dipolar; the mixed diagram where one vertex is magnetic and the other is electric is proportional to $\epsilon_{\mu \nu \rho \lambda} F^{\mu \nu} F^{\rho \lambda}=0$ for photons with equal momenta. The sum of the diagrams produces the following contribution to the photon vacuum-polarization tensor:

$$
\begin{gathered}
\Pi^{\mu \nu}\left(q^{2}\right)=\Pi\left(q^{2}\right)\left(q^{2} g^{\mu \nu}-q^{\mu} q^{\nu}\right) \\
\approx \beta q^{2}\left(q^{2} g^{\mu \nu}-q^{\mu} q^{\nu}\right), \\
\beta=\frac{\mathcal{D}^{2}+\mathcal{M}^{2}}{8 \pi^{2}}\left(1-\frac{1}{3} \ln \frac{m_{\chi}^{2}}{\mu^{2}}\right),
\end{gathered}
$$

where the photon momentum is taken to be small, $q^{2} \ll$ $m_{\chi}^{2}$ (resulting in $\beta q^{2} \ll 1$ ), and $\mu$ is the renormalization scale, which should be smaller than $\Lambda$. We take $\mu \lesssim$ $1 \mathrm{TeV}$ for our estimates. With this self-energy correction, the photon propagator for $\beta q^{2} \ll 1$ can be written as

$$
-i g^{\mu \nu}\left(\frac{1}{q^{2}}-\frac{1}{q^{2}-1 / \beta}\right) \text {. }
$$

The second term above generates a correction to the muon gyromagnetic ratio $\delta a_{\mu}=-\alpha m_{\mu}^{2} \beta / 3 \pi$. Interestingly, this contribution is not explicitly suppressed by the DDM mass. In view of recent measurements [25] and comparison with the SM predictions, we require that $\delta a_{\mu}$ does not exceed $10^{-9}$, whereby $\left(\mathcal{D}^{2}+\mathcal{M}^{2}\right)^{1 / 2}<$

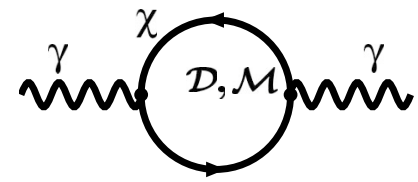

FIG. 5. One-loop correction to the photon self-energy induced by dipole moments $\mathcal{M}, \mathcal{D}$ of the dark-matter particle.

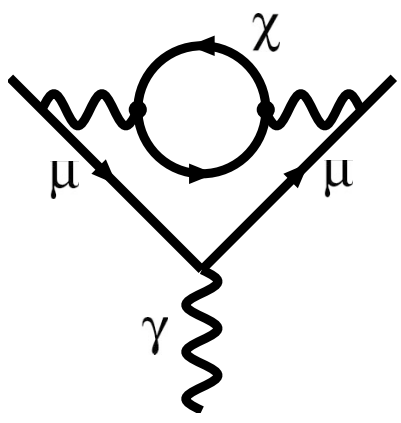

FIG. 6. Lowest-order correction to the muon anomalous magnetic-moment induced by dipole moments of the darkmatter particle.

$6 \times 10^{-15} e \mathrm{~cm}$. The order of magnitude of this result can be obtained on dimensional grounds, if we consider that the DDM dipole moment contributes to $a_{\mu}$ via at least a two-loop graph (see Fig. 6), with two electromagnetic couplings and two dipole couplings. Including a factor $1 / 16 \pi^{2}$ per loop, one obtains the estimate,

$$
\delta a_{\mu} \sim \frac{e^{2}}{\left(16 \pi^{2}\right)^{2}}\left(\mathcal{D}^{2}+\mathcal{M}^{2}\right) \mathcal{E}^{2},
$$

where $\mathcal{E}$ is the characteristic energy scale for the process. In the case of the muon, $\mathcal{E} \sim m_{\mu}$, which reproduces the rigorous result to within an order of magnitude.

\section{B. Electric Dipole Moments}

Furry's theorem tells us that in evaluating radiative corrections to a process one should only keep the diagrams with an even number of photons attached to a closed loop. ${ }^{3}$ Contributions with an odd number of photons attached sum to zero. On the other hand, one must have an odd number of time-reversal-odd (T-odd) EDM vertices in the DDM loop to generate a T-odd operator. These considerations show that the lowest-order nonvanishing diagram must have four photons attached to the DDM loop; diagrams with two photons attached, similar to the one in Fig. 6, vanish (see above). Out of the four photons attached to the DDM loop, either one or three can have EDM coupling to DDM. Note that in this scenario both electric and magnetic DDM moments are necessary to generate a dipole moment for a SM fermion. With these considerations in mind, the lowest-order three-loop diagram that induces an EDM for a charged fermion is shown in Fig. 7. One obtains the following estimate for the induced electric dipole moment:

$$
\mathcal{D}_{f} \sim\left[\mathcal{D} \mathcal{M}\left(\mathcal{D}^{2}+\mathcal{M}^{2}\right)\right] \frac{e^{3} m_{f}^{3}}{\left(16 \pi^{2}\right)^{3}} \ln ^{2} \frac{m_{\chi}}{m_{f}},
$$

\footnotetext{
${ }^{3}$ Since the theorem is valid for interactions that preserve charge conjugation invariance we can apply it to electric and magnetic dipole moment interactions.
} 


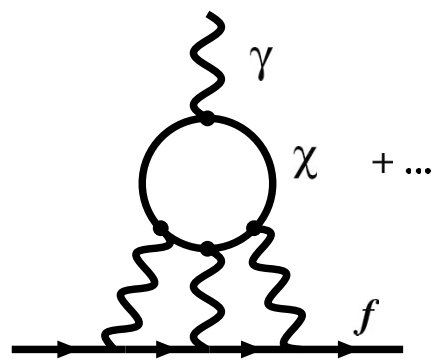

FIG. 7. Three-loop contributions to the EDM of a charged fermion $f$. Either one or three of the DDM-photon interaction vertices must be of EDM type. The dots indicate all other diagrams which can be obtained from the one shown by permutation of the interaction vertices.

where a possible double-logarithmic enhancement is included. For the electron, the present limit is $\mathcal{D}_{e}<4 \times$ $10^{-27} e \mathrm{~cm}$, which implies $(\mathcal{D}, \mathcal{M})<3 \times 10^{-13} e \mathrm{~cm}$ for $m_{\chi} \sim 100 \mathrm{GeV}$ and $\mathcal{D} \sim \mathcal{M}$. For smaller $m_{\chi}$ the limit becomes weaker.

There are constraints on the EDMs of other systems, such as the neutron and the mercury atom. It is nontrivial to translate such constraints into limits on the underlying interaction. In the case of the neutron, one may attempt to treat the neutron as a point particle for virtual-photon energies below $1 \mathrm{GeV}$. For higher loop momenta, photons begin seeing the quarks and the contribution to the EDM becomes suppressed by the quark masses. In this case one may use the above equation $D \sim M$ with $m_{f} \rightarrow m_{n}$ and no logarithmic enhancement, in order to estimate the neutron EDM:

$$
\mathcal{D}_{n}=\left[\mathcal{D} \mathcal{M}\left(\mathcal{D}^{2}+\mathcal{M}^{2}\right)\right] \frac{e^{3}\left|\kappa_{n}\right|^{3} m_{n}^{3}}{\left(16 \pi^{2}\right)^{3}}<6 \times 10^{-26} e \mathrm{~cm}
$$

which results in the limit $(\mathcal{D}, \mathcal{M}) \leqq 4 \times 10^{-15} e \mathrm{~cm}$ (assuming $\mathcal{D} \sim \mathcal{M}$. In the above equation, $\kappa_{n}=-1.91$ is the magnetic-moment of the neutron. It appears because the neutron is neutral, and couples to the photon in Fig. 7 via a magnetic-moment interaction. The limit for the EDM of the mercury atom is much stronger than the neutron, $\mathcal{D}_{H g} \lesssim 10^{-28} e \mathrm{~cm}$. Unfortunately, the mercury atom is a complicated system for which the EDM is influenced by many sources. Therefore, we leave the $\mathrm{Hg}$ limit for future study.

\section{C.W Boson Mass}

The DDM can contribute to the running of the finestructure constant for momenta ranging up to the $Z^{0}$ mass. Such running will affect the relationship between the Fermi constant $G_{F}$, the mass of the $W^{ \pm}$boson, and the fine-structure constant at zero momentum:

$$
m_{W}^{2}=\frac{\pi \alpha}{\sqrt{2} G_{F}} \frac{1}{\left(1-m_{W}^{2} / m_{Z}^{2}\right)(1-\Delta r)},
$$

where $\Delta r$ is a correction calculable in a given theory. The interaction in Eq. (1) modifies the standard expression for $\Delta r$, whereby $\Delta r^{N e w}=\Pi\left(m_{Z}^{2}\right)-\Pi(0)$. In the standardmodel $\Delta r^{S M}=0.0355 \pm 0.0019 \pm 0.0002$. On the other hand, one can use experimentally measured values for $\alpha$, $m_{W, Z}$, and $G_{F}$ in Eq. (14) to infer $\Delta r^{\exp }=0.0326 \pm$ 0.0023 , which gives $\Delta r^{\text {New }}<0.003$ at the $95 \%$ confidence level. Therefore, we obtain the limit $\left(\mathcal{D}^{2}+\mathcal{M}^{2}\right)^{1 / 2} \lesssim$ $3 \times 10^{-16} e \mathrm{~cm}$. A full calculation of the vacuumpolarization yields the constraint shown in Fig. 1. This turns out to be the strongest constraint due to precision tests of the standard-model.

\section{Z-Pole Observables}

The DDM will contribute to various $Z^{0}$-pole observables through two-loop diagrams similar to the one shown in Fig. 8, at the level $\alpha\left(\mathcal{D}^{2}+\mathcal{M}^{2}\right) m_{Z}^{2} / 64 \pi^{3}$. Requiring that these contributions do not exceed the $\sim 0.1 \%$ precision to which $Z^{0}$-pole observables are typically known [26] results in the constraint $\left(\mathcal{D}^{2}+\right.$ $\left.\mathcal{M}^{2}\right)^{1 / 2}<10^{-14} e \mathrm{~cm}$. Note that in order for perturbation theory to apply for energies $\mathcal{E} \sim m_{Z}$, one must have $(\mathcal{D}, \mathcal{M}) m_{Z}<1$, which means $(\mathcal{D}, \mathcal{M}) \lesssim 7 \times$ $10^{-16} e \mathrm{~cm}$. Interestingly, consistency with a perturbative treatment at the $Z^{0}$ pole imposes much stronger constraints on the DDM than the $Z^{0}$-pole observables themselves.

\section{E. Direct Production}

If kinematically allowed, DDM can be directly produced in various scattering and decay experiments. In this case one may use the "missing-energy" signature to constrain the DDM couplings. Here, we consider missing-energy constraints from both low-energy $\left(B^{+}\right.$ and $K^{+}$meson decays) as well as collider (LEP, CDF) experiments.

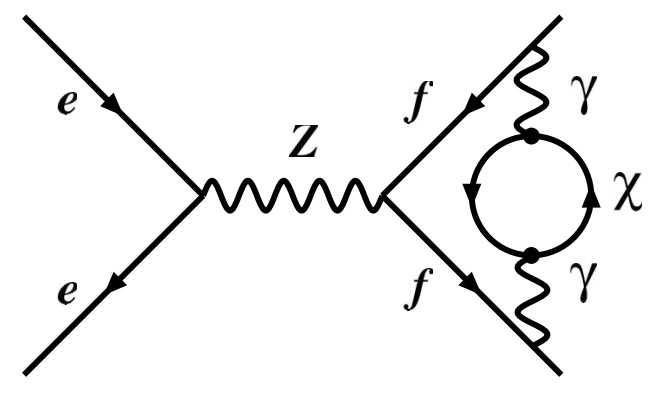

FIG. 8. Lowest-order correction to $Z^{0}$-pole observables induced by dipole moments of the dark-matter particle. 


\section{1. $B^{+}$and $K^{+}$Decays}

Searching for light $\left(m_{\chi} \lesssim 1 \mathrm{GeV}\right)$ dark-matter using missing-energy signatures in rare $B^{+}$meson decays was originally suggested in Ref. [27]. There, data from BABAR [28] and CLEO [29] were used to set a limit $\operatorname{Br}\left(B^{+} \rightarrow K^{+}+\right.$invisible $) \lesssim 10^{-4} \quad$ [derived from $\left.\operatorname{Br}\left(B^{+} \rightarrow K^{+} \bar{\nu} \nu\right)\right]$. This limit can be used to constrain the dipole moments of dark-matter. The diagram for $B^{+} \rightarrow K^{+} \bar{\chi} \chi$ decay is shown in Fig. 9(a). The rate for this decay can be related to the photon-exchange contribution to $\left.B^{+} \rightarrow K^{+} l^{+} l^{-}\right|_{\gamma}$ shown in Fig. 9(b). Since the graphs have identical topologies, the difference in rates will come from the difference in effective couplings and the final-state phase-space integrals. One can estimate,

$$
\begin{aligned}
\frac{B r\left(B^{+} \rightarrow K^{+} \bar{\chi} \chi\right)}{\left.B r\left(B^{+} \rightarrow K^{+} l^{+} l^{-}\right)\right|_{\gamma}} \sim & \frac{\left(\mathcal{D}^{2}+\mathcal{M}^{2}\right) m_{B^{+}}^{2}}{e^{2}} \\
& \times \frac{P S\left(K^{+} \bar{\chi} \chi\right)}{P S\left(K^{+} l^{+} l^{-}\right)},
\end{aligned}
$$

where $P S(\cdots)$ stand for the corresponding final-state phase-space integrals, and $m_{B^{+}}=5.279 \mathrm{GeV}$ is the $B^{+}$ mass. Belle [30] and BABAR [31] find $\mathrm{Br}\left(B^{+} \rightarrow\right.$ $\left.K^{+} l^{+} l^{-}\right) \lesssim 10^{-6}$. Since the ratio of the phase-space integrals is of order unity, and since in the absence of accidental cancellations $\left.\quad B r\left(B^{+} \rightarrow K^{+} l^{+} l^{-}\right)\right|_{\gamma} \lesssim$ $B r\left(B^{+} \rightarrow K^{+} l^{+} l^{-}\right)$, one obtains the constraint

$$
2 \times 10^{-6}\left(\frac{\left(\mathcal{D}^{2}+\mathcal{M}^{2}\right)^{1 / 2} m_{B^{+}}}{e}\right) \lessgtr 10^{-4}
$$

which leads to $\left(\mathcal{D}^{2}+\mathcal{M}^{2}\right)^{1 / 2} \lesssim 3.8 \times 10^{-14} e \mathrm{~cm}$. This constraint is relevant for $m_{\chi}<\left(m_{B^{+}}-m_{K^{+}}\right) / 2=$ $2.38 \mathrm{GeV}$.

Rare $K^{+}$decays can be treated in a similar manner. The relevant branching ratios are $B r\left(K^{+} \rightarrow \pi^{+} e^{+} e^{-}\right)=$ $2.88_{-0.13}^{+0.13} \times 10^{-7}$ and $\operatorname{Br}\left(K^{+} \rightarrow \pi^{+} \bar{\nu} \nu\right)=0.157_{-0.082}^{+0.175} \times$ $10^{-9}$ [26]. The resulting constraint on the dipole moment is $\left(\mathcal{D}^{2}+\mathcal{M}^{2}\right)^{1 / 2} \lesssim 1.5 \times 10^{-15} e \mathrm{~cm}$. This constraint applies for $m_{\chi}<\left(m_{K^{+}}-m_{\pi^{+}}\right) / 2=0.18 \mathrm{GeV}$. We see that constraints from $B^{+}$and $K^{+}$decays are not competitive with other constraints shown in Fig. 1.

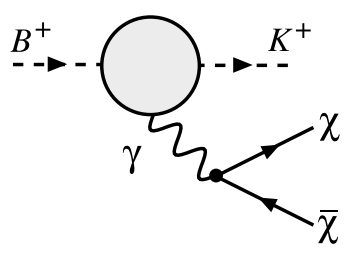

(a)

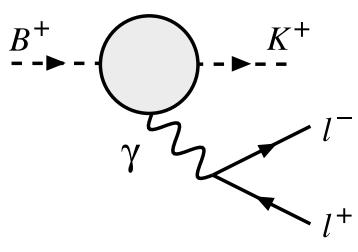

(b)
FIG. 9. Photon-exchange contributions to (a) $B r\left(B^{+} \rightarrow\right.$ $\left.K^{+} \bar{\chi} \chi\right)$ and (b) $\operatorname{Br}\left(B^{+} \rightarrow K^{+} l^{+} l^{-}\right)$. The blobs collectively represent quark flavor-changing interactions.

\section{Collider Experiments}

A typical example of a process where DDM can be directly produced in a collider experiment is shown in Fig. 10. Here, two fermions $f$ scatter to produce a finalstate containing some set of visible particles $X$ (photon, multiple jets, etc.) along with particles that are not detected. In the SM, the latter are neutrinos. Limits on the rate for such processes have been set by, e.g., the L3 and CDF collaborations [32]. At LEP, $X$ consisted of a single photon whereas at CDF it consisted of one or more hadronic jets.

In order to translate constraints from collider experiments into limits on DDM couplings one needs an analytical expression for the rate for $f \bar{f} \rightarrow X \bar{\chi} \chi$. Naive application of the effective Lagrangian in Eq. (1) would result in upper limits from these missing-energy searches of roughly $10^{-17} \mathrm{e} \mathrm{cm}$. However, this constraint does not actually exclude larger values of the dipole moments. Indeed, as discussed above, perturbation theory will break down when the energy scale for the process $\mathcal{E}$ satisfies $(\mathcal{D}, \mathcal{M}) \mathcal{E} \gtrsim 1$. This means that missing-energy searches from L3 $(\mathcal{E} \approx 200 \mathrm{GeV})$ and $\mathrm{CDF}(\mathcal{E}=$ $1.8 \mathrm{TeV}$ ) cannot be used to probe effective dipole moments $(\mathcal{D}, \mathcal{M})>10^{-16} e \mathrm{~cm}$ and $(\mathcal{D}, \mathcal{M})>10^{-17} e \mathrm{~cm}$, respectively, unless the underlying physics that gives rise to the dipole moment is specified.

\section{F. Other Laboratory Constraints}

Important constraints can be obtained for millicharged particles from the Lamb shift $[10,33]$ and from a targeted experiment at SLAC [34]. We have checked, however, that due to the different energy dependence of the photondipole vertex, as opposed to the photon-millicharge vertex, the DDM-induced correction to the Lamb shift is small for dipole moments not eliminated by other precision measurements, such as the running of the finestructure constant. Likewise, although the SLAC experiment is in principle sensitive to neutral particles with a dipole, the production and energy deposition of dipole particles is sufficiently small, for dipole moments consis-

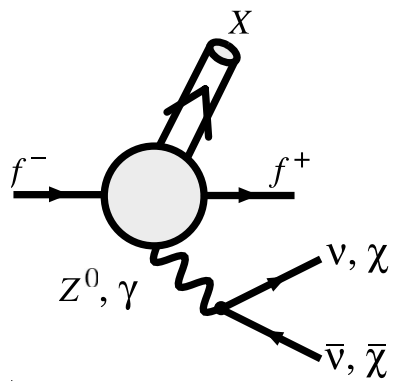

FIG. 10. A typical process that would produce a missingenergy signature in a collider experiment. Here, $X$ stands for the visible portion of the final-state. Neutrinos or DDM may carry a large fraction of the energy but are not detected. 
tent with accelerator experiments, to evade detection in the SLAC experiment.

\section{CONSTRAINTS FROM LARGE-SCALE STRUCTURE AND THE CMB}

We now consider the effects of the interaction $\mathcal{L}_{\gamma \chi}$ on the evolution of cosmological perturbations and their resulting imprints on the matter power spectrum and the CMB. A dipole moment can induce a coupling of the dark-matter to the baryon-photon fluid by scattering from photons through the diagrams shown in Fig. 11, or by scattering from protons, helium nuclei, and/or electrons through the diagram shown in Fig. 4. What we will show below is that the dark-matter is coupled to the baryon-photon fluid at early times, and decouples at later times. When the dark-matter is coupled to the photonbaryon fluid, the pressure of the plasma resists the growth of gravitational potential wells. Thus, the shortwavelength modes of the density field that enter the horizon at early times will have their growth suppressed relative to the standard calculation resulting in a suppression of small-scale power. The evolution of the longerwavelength modes that enter the horizon after the darkmatter has decoupled remain unaffected. Before presenting the results of our detailed analysis, we begin with some simple estimates.

We first show that DDM-photon scattering is negligible compared with DDM-baryon scattering in providing the drag-force between the DDM fluid and the baryon-photon fluid. To do so, we first estimate the drag-force per unit mass (i.e., the deceleration) on a DDM-particle that moves with a velocity $V$ with respect to the rest frame of a blackbody at temperature $T$. The diagrams in Fig. 11 will lead to a photon-DDM scattering cross-section $\sigma_{\chi \gamma} \sim\left(\mathcal{D}^{4}+\mathcal{M}^{4}\right) E_{\gamma}^{2}$. Considering that the momentum transfer to the DDM-particle in each scatter is $\sim E_{\gamma}$ and that the difference of the fluxes of photons moving in the same versus opposite direction to the DDM-particle is $\sim T^{3}(\mathrm{~V} / \mathrm{c})$, we conclude that the deceleration due to photon scattering is $a_{\gamma} \sim\left(\mathcal{D}^{4}+\mathcal{M}^{4}\right) T^{6}(V / c) / m_{\chi}$.

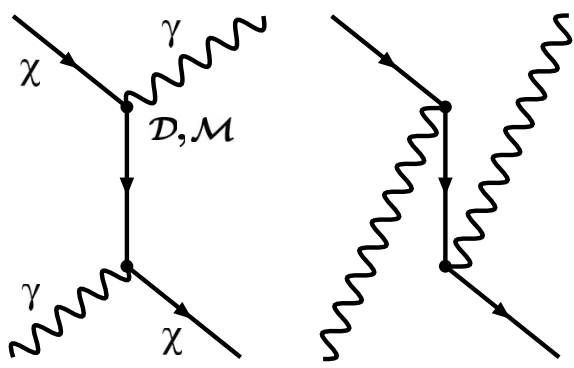

FIG. 11. The photon-DDM scattering diagram, the analogue of the Compton-scattering diagrams for electric or magnetic moments.
We next estimate $a_{b}$, the drag-force per unit mass due to DDM-proton scattering. We first note that the peculiar velocity of the baryon-photon fluid (obtained from the continuity equation) in the early Universe will be $V \sim$ $(H / k) c \delta$, where $H$ is the Hubble parameter, $k$ is the physical wavenumber of the mode in question, and $\delta \sim$ $10^{-5}$ is the amplitude of the fractional density perturbation. Since $(H / k) \lesssim 1$ for modes inside the horizon, we must have $V \lesssim 10^{-5} \mathrm{c}$. On the other hand, the proton thermal velocity dispersion is $\bar{v}_{p} \sim\left(T / m_{p}\right)^{1 / 2} c \gtrsim$ $10^{-4.5} c$ before recombination. Thus, for the early times of interest to us here, the relative velocity between the DDM and the baryon-photon fluid is small compared with the thermal proton velocities. Thus, the appropriate relative velocity to use in Eq. (5) in estimating the protonDDM cross-section is $\bar{v}_{p}$, resulting in a DDM-proton cross-section $\sigma_{\chi b} \sim e^{2}\left(\mathcal{D}^{2}+\mathcal{M}^{2}\right) / \bar{v}_{p}^{2}$. The momentum transfer per scatter is $\sim \mu \bar{v}_{p}$, where $\mu$ is the proton-DDM reduced mass, and the difference of the fluxes of protons moving with as opposed to against the DDM fluid is $n_{p} V$, where $n_{p}$ is the proton density. The drag-force per unit mass on the DDM fluid due to scattering with protons is thus $a_{b} \sim e^{2}\left(\mathcal{D}^{2}+\mathcal{M}^{2}\right)\left(\mu / m_{\chi}\right)\left(V / \bar{v}_{p}\right) n_{p}$. We also conclude from the appearance of $\mu$ in this result that drag due to scattering from electrons is negligible compared with baryon drag.

Since $n_{p} \propto T^{3}$ and $\bar{v}_{p} \propto T^{1 / 2}$, we find $a_{b} \propto T^{2.5}$ as opposed to $a_{\gamma} \propto T^{6}$. Thus, at early times, photon drag dominates while baryon drag dominates at later times. The transition occurs at a temperature $T \sim \mathrm{GeV}$ for values of $m_{\chi}$ and $(\mathcal{D}, \mathcal{M})$ of interest to us, and such high temperatures correspond to (comoving) horizon scales considerably smaller than the distance scales $(\gtrsim$ Mpc) probed by large-scale structure. We can thus neglect photon drag. From $a_{b} \propto T^{2.5}$ we infer a deceleration time for the DDM fluid $t_{\mathrm{dec}}=V / a_{b} \propto T^{-2.5}$. Since this decreases more rapidly than the Hubble time $t_{U} \sim m_{\mathrm{Pl}} T^{-2}$ (where $m_{\mathrm{Pl}} \sim 10^{19} \mathrm{GeV}$ is the Planck mass), we conclude that DDM particles are tightly coupled to the plasma at early times and then are decoupled at later times. With these rough estimates, the transition temperature is $T \sim$ $10 \operatorname{keV}\left(\mathcal{D}_{15}^{2}+\mathcal{M}_{15}^{2}\right)^{-2}\left(1+m_{\chi} / m_{p}\right)^{2}$ suggesting that power on scales smaller than $\lambda \sim 10^{-2}\left(\mathcal{D}_{15}^{2}+\mathcal{M}_{15}^{2}\right)^{2} \times$ $\left(1+m_{\chi} / m_{p}\right)^{-2} \mathrm{Mpc}$ will be suppressed. The $T^{0.5}$ dependence of the ratio of the deceleration and expansion times suggests furthermore that the small-scale suppression will change gradually, rather than exponentially, with wavenumber $k$. Knowing that the linear-theory power spectrum is measured and roughly consistent with scale invariance down to distances $\sim \mathrm{Mpc}$ leads us to conclude that dipole moments $\left(\mathcal{D}^{2}+\mathcal{M}^{2}\right)^{1 / 2} \gtrsim 5 \times 10^{-15}(1+$ $\left.m_{\chi} / m_{p}\right)^{1 / 2} e \mathrm{~cm}$ will be ruled out. Strictly speaking, when $m_{\chi}<m_{p}$, the detailed calculation must take into account the velocity dispersion of the DDM particles; our 
detailed calculation below includes these effects. As seen below, the detailed analysis leads to a slightly stronger constraint.

\section{A. Exact Equations}

The standard calculation of perturbations in an expanding universe requires the solution of the combined Einstein and Boltzmann equations for the distribution functions of the dark-matter, baryons, photons, and neutrinos including all relevant standard-model interactions (see, e.g., Refs. [35,36] and references therein). Since the perturbations are initially very small, linear perturbation theory is an excellent approximation; this allows us to solve the perturbation equations in Fourier space at each wavenumber $k$ independently of all other wavenumbers (modes are uncoupled). The scattering of photons and baryons by DDM through the interaction $\mathcal{L}_{\gamma \chi}$ influences the growth of cosmological perturbations by introducing additional collision terms to the Boltzmann equations, which ultimately result in a drag-force between the DDM and the colliding species in the equations describing the cosmological fluid (see, e.g., Refs. [37,38], which consider similar effects). Below we present the exact perturbation equations including the effects of dark-matter with electric or magnetic dipole moments. Since solutions to these equations are numerically intensive when photons and baryons are tightly coupled through Compton-scattering, we also discuss the equations appropriate for solving for the DDM, photon, and baryon perturbations during the epoch of tight coupling.

In the synchronous gauge the equations describing the evolution of baryons, photons, and dark-matter with an electric or magnetic dipole moment are

$$
\begin{aligned}
& \dot{\delta}_{\gamma}=-\frac{4}{3} \theta_{\gamma}-\frac{2}{3} \dot{h}, \quad \dot{\delta}_{b}=-\theta_{b}-\frac{1}{2} \dot{h}, \quad \dot{\delta}_{\chi}=-\theta_{\chi}-\frac{1}{2} \dot{h}, \\
& \dot{\theta}_{\gamma}=k^{2}\left(\frac{1}{4} \delta_{\gamma}-\Sigma_{\gamma}\right)+a n_{e} \sigma_{T}\left(\theta_{b}-\theta_{\gamma}\right)+a n_{\chi}\langle\sigma\rangle_{\chi \gamma}\left(\theta_{\chi}-\theta_{\gamma}\right), \\
& \dot{\theta}_{b}=-\frac{\dot{a}}{a} \theta_{b}+c_{s b}^{2} k^{2} \delta_{b}+\frac{4 \rho_{\gamma}}{3 \rho_{b}} a n_{e} \sigma_{T}\left(\theta_{\gamma}-\theta_{b}\right)+a n_{\chi}\langle\sigma v\rangle_{\chi b}\left(\theta_{\chi}-\theta_{b}\right), \\
& \dot{\theta}_{\chi}=-\frac{\dot{a}}{a} \theta_{\chi}+c_{s \chi}^{2} k^{2} \delta_{\chi}+\frac{\rho_{b}}{\rho_{\chi}} a n_{\chi}\langle\sigma v\rangle_{\chi b}\left(\theta_{b}-\theta_{\chi}\right)+\frac{4 \rho_{\gamma}}{3 \rho_{\chi}} a n_{\chi}\langle\sigma\rangle_{\chi \gamma}\left(\theta_{\gamma}-\theta_{\chi}\right) .
\end{aligned}
$$

While the evolution equations for the density contrast $\delta_{j}=\delta \rho_{j} / \rho_{j}$ for each species $j \in\{\gamma, b, \chi\}$ are as in the standard case [35], as discussed above, the evolution equations for the fluid-velocity perturbations have additional drag-force terms due to the photon-DDM interaction. Note that in these equations and what follows the variable $\theta_{j}=i k V_{j}$ is the divergence of the fluid-velocity in Fourier space, $\Sigma_{j}$ is the shear, $c_{s j}$ is the intrinsic sound speed, and $n_{j}$ and $\rho_{j}$ are the background number and energy densities of a particular species $j$, respectively. The variable $h$ is the trace of the scalar metric perturbation in Fourier space (not to be confused with the Hubble parameter), $a$ is the cosmological scale factor, and an overdot represents a derivative with respect to the conformal time $\tau$. Furthermore, $\sigma_{T}$ is the Thomson crosssection, while

$$
\langle\sigma\rangle_{\chi \gamma}=\frac{80}{21} \pi\left(\mathcal{D}^{4}+\mathcal{M}^{4}\right) T_{\gamma}^{2},
$$

is the appropriately thermally-averaged DDM-photon cross-section, which can be obtained from the differential cross-section $[39,40]$,

$$
\frac{d \sigma_{\chi \gamma}}{d \Omega}=\frac{\left(\mathcal{D}^{4}+\mathcal{M}^{4}\right) E_{\gamma}^{2}}{8 \pi^{2}}\left(3-\cos ^{2} \theta\right)
$$

for photon-DDM scattering. As argued above, the
photon-DDM drag term is small, and we consider it no further in Eq. (17).

The quantity

$$
\langle\sigma v\rangle_{\chi b}=\frac{4(1+\xi Y)}{3 \pi^{2} \sqrt{\left\langle v_{p}\right\rangle^{2}+\left\langle v_{\chi}\right\rangle^{2}}} \frac{m_{\chi}}{m_{\chi}+m_{\mathrm{p}}} e^{2}\left(\mathcal{D}^{2}+\mathcal{M}^{2}\right)
$$

is the appropriate thermally-averaged cross-section times relative velocity for the baryon-DDM coupling, and

$$
\xi=8 \frac{m_{\chi}+m_{\mathrm{p}}}{m_{\chi}+4 m_{\mathrm{p}}} \sqrt{\frac{\left\langle v_{p}\right\rangle^{2}+\left\langle v_{\chi}\right\rangle^{2}}{\left\langle v_{p}\right\rangle^{2}+4\left\langle v_{\chi}\right\rangle^{2}}}-1
$$

is the relative efficiency for coupling to helium nuclei compared to protons. The Appendix provides a derivation of this collision coefficient. In these expressions, $Y=$ $\rho_{\mathrm{He}} / \rho_{b} \simeq 0.24$ is the cosmological helium mass fraction (approximating $\left.m_{H e} \simeq 4 m_{p}\right),\left\langle v_{p}\right\rangle=\sqrt{8 T_{b} / \pi m_{p}}$ is the average thermal speed of the protons, $\left\langle v_{\chi}\right\rangle=$ $\sqrt{8 T_{\chi} / \pi m_{\chi}}$ is the average thermal speed of the DDM, and $T_{\gamma}, T_{b}$, and $T_{\chi}$ are the photon, baryon, and DDM temperatures, respectively. The dark-matter temperature evolves according to 


$$
\begin{aligned}
\dot{T}_{\chi}= & -2 \frac{\dot{a}}{a} T_{\chi}+\frac{2 a \rho_{b}\langle\sigma v\rangle_{\chi b}^{\mathrm{T}}}{m_{\chi}+m_{p}}\left(T_{b}-T_{\chi}\right) \\
& +\frac{8 a \rho_{\gamma}\langle\sigma\rangle_{\chi \gamma}}{3 m_{\chi}}\left(T_{\gamma}-T_{\chi}\right),
\end{aligned}
$$

where $\langle\sigma v\rangle_{\chi b}^{\mathrm{T}}$ is the same as the expression given in Eq. (20) with the replacement of $\xi$ by $\xi^{\mathrm{T}}$ which is given by the expression in Eq. (21) with the factor $\left(m_{\chi}+\right.$ $\left.m_{p}\right) /\left(m_{\chi}+4 m_{p}\right) \quad$ replaced by $\left[\left(m_{\chi}+m_{p}\right) /\left(m_{\chi}+\right.\right.$ $\left.\left.4 m_{p}\right)\right]^{2}$. The final term, describing the dark-matter heating by photons, is important at very early times. For the dipole strength and mass range considered, the influence is manifest only on very small length scales, below the range of interest.

At early times, the DDM temperature $T_{\chi} \simeq T_{b}$, but at later times, when the DDM decouples, $T_{\chi}$ drops relative to $T_{b}$. The DDM-proton cross-section is $\propto v^{-2}$, which leads to $\langle\sigma v\rangle_{\chi b} \propto\left\langle v_{p}\right\rangle^{-1}$. As a result, we cannot directly apply the results of Ref. [37], wherein a velocity-independent dark-matter-baryon interaction was assumed. However, we have verified that we recover their results if we take a velocity-independent cross-section as the source of darkmatter-baryon drag.

\section{B. Tightly Coupled Equations}

At early times when $\tau_{c}^{-1} \equiv a n_{e} \sigma_{T} \gg \dot{a} / a$ the rapid scattering of baryons and photons forces these species to have nearly equal fluid velocities, and consequently the solution of the equations shown in Eq. (17) is numerically intensive. Following standard procedures $[35,41]$ we derive a set of equations to leading order in the (conformal) Compton-scattering time $\tau_{c}$ that are appropriate for evolving the fluid variables through this epoch of tight coupling. We first write down an equation for the time derivative of $\theta_{b}-\theta_{\gamma}$ which is usually termed the baryonphoton "slip" to leading order in $\tau_{c}$,

$$
\begin{aligned}
\dot{\theta}_{b}-\dot{\theta}_{\gamma}= & \frac{2 R_{\gamma b}}{1+R_{\gamma b}} \frac{\dot{a}}{a}\left(\theta_{b}-\theta_{\gamma}\right)+\frac{\tau_{c}}{1+R_{\gamma b}} \\
& \times\left[-\frac{\ddot{a}}{a} \theta_{b}+k^{2}\left(c_{s b}^{2} \dot{\delta}_{b}-\frac{1}{4} \dot{\delta}_{\gamma}-\frac{1}{2} \frac{\dot{a}}{a} \delta_{\gamma}\right)\right. \\
& \left.+\frac{1}{\tau_{\chi}}\left(\dot{\theta}_{\chi}-\dot{\theta}_{b}\right)-\frac{1}{2} \frac{\dot{a}}{a} \frac{1}{\tau_{\chi}}\left(\theta_{\chi}-\theta_{b}\right)\right],
\end{aligned}
$$

where we have introduced the (conformal) DDM-baryon scattering time $\tau_{\chi}^{-1} \equiv a n_{\chi}\langle\sigma v\rangle_{\chi b}$, and $R_{\gamma b} \equiv 4 \rho_{\gamma} /\left(3 \rho_{b}\right)$. It is useful to separate this equation as a sum of the terms not containing $\tau_{\chi}$ (this is just the time derivative of the standard slip, which we denote $S_{b \gamma}$ ), and the new terms introduced by the DDM coupling,

$$
\dot{\theta}_{b}-\dot{\theta}_{\gamma}=\dot{S}_{b \gamma}+\beta\left[\left(\dot{\theta}_{\chi}-\dot{\theta}_{b}\right)-\frac{1}{2} \frac{\dot{a}}{a}\left(\theta_{\chi}-\theta_{b}\right)\right],
$$

where

$$
\beta=\frac{1}{1+R_{\gamma b}} \frac{\tau_{c}}{\tau_{\chi}}
$$

is the parameter that controls how strongly the new interaction affects the evolution of the slip. In terms of these definitions the baryon-velocity evolution equation is

$$
\begin{aligned}
\dot{\theta}_{b}= & \frac{1}{1+R_{\gamma b}+\beta R_{\gamma b}} \\
& \times\left(-\frac{\dot{a}}{a} \theta_{b}+k^{2}\left[c_{s b}^{2} \delta_{b}+R_{\gamma b}\left(\frac{1}{4} \delta_{\gamma}-\Sigma_{\gamma}\right)\right]\right. \\
& \left.+R_{\gamma b}\left\{\dot{S}_{b \gamma}+\beta\left[\dot{\theta}_{\chi}-\frac{1}{2} \frac{\dot{a}}{a}\left(\theta_{\chi}-\theta_{b}\right)\right]\right\}\right) .
\end{aligned}
$$

The photon-evolution equation is then given by the exact expression

$$
\begin{aligned}
\dot{\theta}_{\gamma}= & -\frac{1}{R_{\gamma b}}\left[\dot{\theta}_{b}+\frac{\dot{a}}{a} \theta_{b}-c_{s b}^{2} k^{2} \delta_{b}-\frac{1}{\tau_{\chi}}\left(\theta_{\chi}-\theta_{b}\right)\right] \\
& +k^{2}\left(\frac{1}{4} \delta_{\gamma}-\Sigma_{\gamma}\right) .
\end{aligned}
$$

We use these equations to follow the initial evolution of the baryon and photon fluid variables and switch to the exact equations of Eq. (17) at later times. For the evolution of the DDM fluid variables we always use the exact form of Eq. (17).

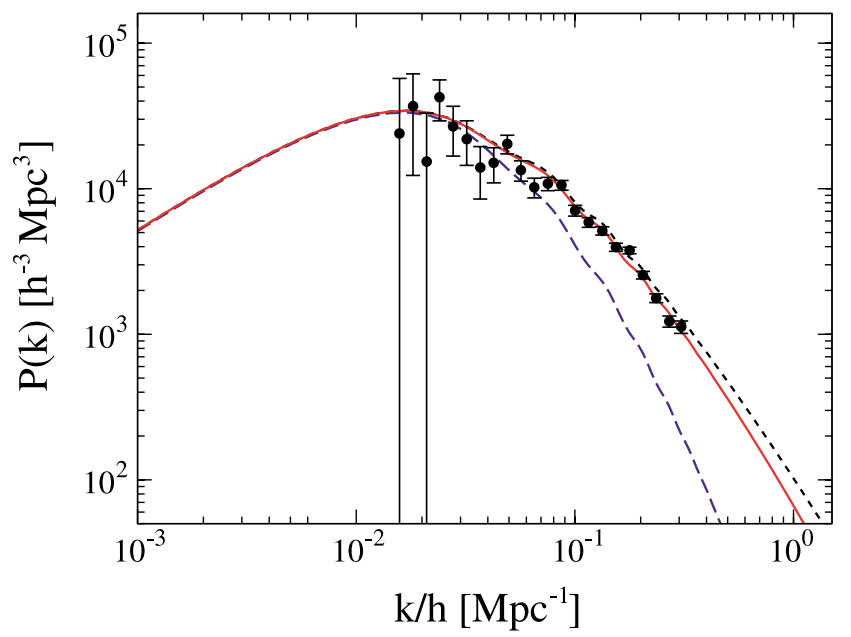

FIG. 10 (color online). Matter power spectra including baryon-DDM drag. The solid curve is for is for $\left(\mathcal{D}^{2}+\right.$ $\left.\mathcal{M}^{2}\right)^{1 / 2}=1.4 \times 10^{-15} e \mathrm{~cm}$ The short-dash is for $\left(\mathcal{D}^{2}+\right.$ $\left.\mathcal{M}^{2}\right)^{1 / 2}=1.0 \times 10^{-16} e \mathrm{~cm}$ The long-dash curve is for $\left(\mathcal{D}^{2}+\right.$ $\left.\mathcal{M}^{2}\right)^{1 / 2}=5 \times 10^{-15} e \mathrm{~cm}$. These are all for a mass of $1 \mathrm{GeV}$. The curves are all for the standard concordance cosmological parameters, and the data points are from SDSS [43]. 


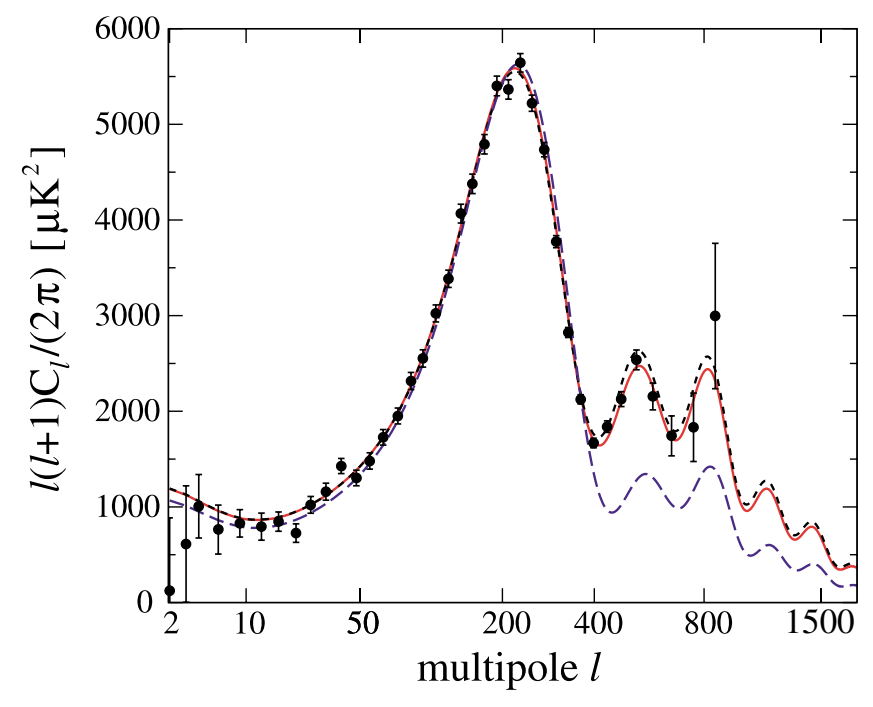

FIG. 11 (color online). CMB power spectra including DDMbaryon drag. The labeling of the curves is the same as in Fig. 10, and the data points are those from WMAP [44].

\section{Effects on the Matter and CMB Power Spectra}

In Fig. 10 we show the linear matter power spectrum and in Fig. 11 we show the angular power spectrum of the $\mathrm{CMB}$ for several values of the dipole moment and for DDM mass $m_{\chi}=1 \mathrm{GeV}$. Physically, the effects of DDM on the matter power spectrum and CMB can be simply understood. Prior to matter-radiation equality the photons have a much larger density than the baryons or the DDM and so to a first approximation completely drive the behavior of the baryon perturbations through Comptonscattering. In turn, the baryon perturbations drive the behavior of the DDM perturbations, very efficiently before DDM decoupling so that the DDM density contrast $\delta_{\chi}$ on scales that enter the horizon during this epoch track the oscillations of the baryon-photon fluid before growing, and less efficiently after DDM decoupling so that the baryons simply cause a drag on the growth of $\delta_{\chi}$. In either case the matter power spectrum is suppressed relative to the standard case. The behavior of the CMB angular power spectrum can be similarly understood. Roughly speaking, the coupling of the DDM and baryons increases the effective baryon loading of the plasma at early times so that the CMB power spectra look similar to those from high-baryon models. This is of course an imperfect correspondence as modes of larger wavelength enter the horizon when the coupling is weaker, and so at later and later times the evolution of the photon perturbations becomes more and more like the standard-CDM case. But due to geometrical projection effects modes of wavenumber $k$ contribute to all $l \lesssim k d_{A}$ where $d_{A}$ is the angular-diameter distance to the last-scattering surface, and so the effects of DDM on small length scales can be noticed even on relatively large angular scales in the CMB.
As the effect of DDM on the CMB may be partially degenerate with other cosmological parameters, we have explored a parameter space that allows us to constrain $m_{\chi}$ and $(\mathcal{D}, \mathcal{M})$ after marginalizing over other cosmological parameters. We consider flat $\Lambda \mathrm{CDM}$ models and our chosen parameter space is the dark-matter density $\Omega_{m} h^{2}$, the baryon density $\Omega_{b} h^{2}$, the Hubble parameter $h$ in units of $100 \mathrm{~km} \mathrm{sec}^{-1} \mathrm{Mpc}^{-1}$, the optical depth $\tau_{\mathrm{CMB}}$ to the last-scattering surface, and the primordial spectral index $n$. We have employed the Markov chain Monte Carlo technique (see, e.g., Ref. [42]) to efficiently explore this parameter space, taking the most recent results from SDSS [43], WMAP [44], CBI [45], VSA [46], and SNe Ia [47] as our data. Note that although DDM has no effect on observations of Type Ia supernovae, we include these data because the other parameters we allow to vary are constrained by these observations. We conclude using a relative-likelihood test that cosmological measurements lead to the bound shown in Fig. 1. The numerical calculations confirm the qualitative behavior discussed above. Dipole moments as large as $\left(\mathcal{D}^{2}+\mathcal{M}^{2}\right)^{1 / 2} \sim 10^{-17} e \mathrm{~cm}$, near the upper end of our allowed parameter space, are thus cosmologically viable.

\section{GAMMA RAYS}

DDM particles in the Galactic halo can annihilate to two photons through the diagrams shown in Fig. 3. Since halo particles move with velocities $v \simeq 300 \mathrm{~km} \mathrm{sec}^{-1} \ll$ $c$, the photons produced will be very nearly monoenergetic with energies equal to the DDM-particle rest mass. The intensity at Earth of such gamma rays is obtained by integrating the emissivity, $n_{\chi}^{2}\left\langle\sigma_{\bar{\chi} \chi} \rightarrow 2 \gamma v\right\rangle$, where $n_{\chi}$ is the DDM number density, along the given line of sight. The intensity is largest toward the Galactic center, where the dark-matter density is largest. In this direction, the gamma-ray intensity is then [4],

$$
\frac{d \mathcal{F}}{d \Omega}=1.0 \times 10^{-10} \frac{\sigma_{\bar{\chi} \chi \rightarrow 2 \gamma} v}{10^{-30} \mathrm{~cm}^{3} \sec ^{-1}} m_{\mathrm{GeV}}^{-2} I \mathrm{~cm}^{-2} \mathrm{sec}^{-1} \mathrm{sr}^{-1},
$$

where $I$ is a scaled integral of $n_{\chi}^{2}$ along a line of sight toward the Galactic center. The numerical coefficient is one-half that from Ref. [4] since we have here particleantiparticle annihilation rather than Majorana annihilation. Roughly speaking, $I \simeq 3-30$ for cored-isothermalsphere models of the Galactic halo, while $I$ can extend up to $\sim 300$ for Navarro-Frenk-White profiles [48]; i.e., uncertainty in the dark-matter distribution in the inner Galaxy leads to an uncertainty of 2 orders of magnitude in the predicted flux. We thus expect

$$
\begin{aligned}
\frac{d \mathcal{F}}{d \Omega}= & \left(3 \times 10^{-13}-3 \times 10^{-11}\right)\left(\mathcal{D}_{17}^{4}\right. \\
& \left.+\mathcal{M}_{17}^{4}\right) \mathrm{cm}^{-2} \mathrm{sec}^{-1} \mathrm{sr}^{-1}
\end{aligned}
$$


To constrain dipole moments from nonobservation of a gamma-ray line, we choose to use the most conservative estimate, $I \simeq 3$ for the dimensionless line integral. Moreover, we are not aware of any EGRET analysis that places limits, in particular, to a line-flux. We thus obtain very conservative limits by using the binned continuum fluxes for the total gamma-ray flux listed in Table 1 of Ref. [48] and noting that a line-flux in that bin cannot exceed the measured continuum flux. The EGRET limits apply for masses $0.1 \lesssim\left(m_{\chi} / \mathrm{GeV}\right) \lesssim 10$, and range from $\mathcal{D}_{17} \lesssim 180$ for $m_{\chi} \lesssim \mathrm{GeV}$ to $\mathcal{D}_{17} \lesssim 100$ for $m_{\chi} \simeq$ $10 \mathrm{GeV}$, as shown in Fig. 1.

Again, a few caveats are in order. First of all, our limit is quantitatively conservative - we chose the halo model that produces the lowest flux, and a detailed EGRET analysis would probably yield a line-flux limit lower than what we have assumed. On the other hand, the strong dependence $\propto \mathcal{D}^{4}$ of the predicted flux on the dipole moment guarantees that the upper limit to the acceptable dipole moment will not depend quite so strongly on these details. Second, if $\mathcal{D}_{17} \gtrsim 5$ in the mass range $100 \mathrm{MeV}$ to $1 \mathrm{GeV}$, then the correct cosmological abundance most likely requires a particle-antiparticle asymmetry. If so, then the annihilation rate in the halo could be reduced far below the values we have obtained above. We conclude by noting that with the increased sensitivity of the GammaRay Large Area Space Telescope (GLAST), a detailed search for a line-flux, and the possibility that the actual halo model provides a more generous annihilation rate, an observable GLAST signature may exist for masses $0.1-1 \mathrm{GeV}$ and dipole moments as low as $\mathcal{D}_{17} \sim 10$.

\section{DISCUSSION}

In this paper we have considered the cosmology and phenomenology of dark-matter particles with a nonzero magnetic or electric dipole moment. We have found that information from precision tests of the standard-model, direct dark-matter searches, gamma-ray experiments, and the $\mathrm{CMB}$ and large-scale structure restrict the dipole moment to be $(\mathcal{D}, \mathcal{M}) \lesssim 3 \times 10^{-16} e \mathrm{~cm}$ for masses $m_{\chi} \lesssim$ few $\mathrm{GeV}$ and $(\mathcal{D}, \mathcal{M}) \lesssim 10^{-24} e \mathrm{~cm}$ for larger masses. (This improves on an earlier limit on WIMP electromagnetic dipole moments based on direct-detection [49].) Some of the allowed regions of parameter space may soon be probed with GLAST and with future more sensitive direct-detection experiments. The electromagnetic interactions of these particles with nuclei are coherent. Moreover, these particles cannot annihilate directly to neutrinos. Therefore, searches for energetic neutrinos from decays of the products of $\bar{\chi} \chi$ annihilation in the Sun or Earth are thus likely to provide less sensitive probes than direct searches [50]. Moreover, if there is a particle-antiparticle asymmetry, then the energeticneutrino flux could be reduced without altering the direct-detection rate.
We have restricted our attention to particles with masses $m_{\chi} \gtrsim \mathrm{MeV}$, with the notion that lower-mass particles will violate BBN limit, as discussed toward the end of Section 2. We also consider masses $m_{\chi} \geqslant$ $\mathrm{MeV}$, as particles of lower-mass will almost certainly undergo relativistic freeze-out and thus lead to unacceptable dark-matter candidates. However, as also noted above that if an $m_{\chi} \lesssim \mathrm{MeV}$ particle has a dipole moment $(\mathcal{D}, \mathcal{M}) \lesssim 10^{-22} e \mathrm{~cm}$ and no other interactions with ordinary matter, then it might still be consistent with BBN. Of course, such a particle will, assuming standard freezeout, have a mass density many orders of magnitude larger than the dark-matter density. But suppose we were to surmise that the dark-matter density was fixed by some other mechanism; e.g., suppose the dipole was sufficiently weak that it never came into equilibrium. In this case, an additional constraint to the dark-matter dipoles can be obtained from energy-loss arguments applied to stars in globular clusters. Such arguments eliminate dipole moments $(\mathcal{D}, \mathcal{M}) \lesssim 6 \times 10^{-23} e \mathrm{~cm}$ for masses $m_{\chi} \lesssim 5 \mathrm{keV}$ [51]. We have also considered constraints from astrophysical phenomena such as the stability of the Galactic disk, lifetime of compact objects, and annihilations in the solar neighborhood [6], and find that these constraints on the mass and interaction strengths are not competitive with those presented here.

It would be of interest to attempt to embed this scenario in a consistent particle-physics model. We might find links between baryonic and nonbaryonic matter abundances, the dark-matter electric dipole moment and the $C P$ violation needed for baryogenesis, and the magnetic moments of dark-matter and baryons. (E.g., Ref. [52] considered a fermionic technibaryon with electromagnetic dipole interactions as a dark-matter candidate.) However, such model building is beyond the scope of the present study. Our approach throughout has been entirely phenomenological, as we have been motivated by the desire to answer the question, "How dark is dark?"

\section{ACKNOWLEDGMENTS}

We thank S. Golwala, J. Albert, M. Zaldarriaga, and R. Shrock for useful discussions. K. S. acknowledges the support of the Canadian NSERC. This work was supported in part by NASA NAG5-9821 and DoE DE-FG0392-ER40701 (at Caltech) and NSF PHY-0099543 (at Dartmouth). M. D. and R. C. thank Caltech for hospitality during the course of this investigation.

\section{APPENDIX: DERIVATION OF THE BARYON- DARK-MATTER COLLISION TERM}

To determine how the cosmological perturbation equations for baryons and dark-matter are altered when we bestow the dark-matter with a magnetic or electric dipole moment, we must formally evaluate the collision operator 
of the general-relativistic Boltzmann equation in a given gauge $[14,35,36]$ for the dipole interaction of Eq. (1). We have completed this calculation in detail, and find that the dipole interaction produces a drag-force proportional to the relative velocity $V=v_{\chi}-v_{b}$ of the dark-matter and baryon fluids. As the relative velocity is gauge invariant in linear perturbation theory and all scatterings are local processes, we may thus take a simpler, more physically transparent approach and just evaluate this drag-force using nonrelativistic statistical mechanics. It is this approach we now present.

We wish to calculate the drag-force per unit mass, or deceleration, due to collisions with protons to the darkmatter fluid as it passes through the baryon-photon fluid. Comoving scales $\lambda \gtrsim$ Mpc enter the horizon when the cosmological temperature is $T \lesssim 10 \mathrm{eV}$, when the DDM particles (which are restricted to $m_{\chi} \gtrsim \mathrm{MeV}$ ) are nonrelativistic. We may thus consider thermal velocity distributions for nonrelativistic baryons and dark-matter. Since the drag-force can only depend on the dark-matterbaryon relative velocity, we take the baryon fluid to be at rest and the dark-matter fluid to have a velocity of magnitude $V$ in the $\hat{x}$ direction. Then, the proton phase-space distribution is

$$
f_{p}\left(\vec{v}_{p}\right)=\frac{n_{p}}{(2 \pi)^{3 / 2} \bar{v}_{p}^{3}} e^{-v_{p}^{2} / 2 \bar{v}_{p}^{2}},
$$

where $\bar{v}_{p}=\left(k T_{p} / m_{p}\right)^{1 / 2}$ is the proton velocity dispersion and $n_{p}$ the proton number density, and

$$
f_{\chi}\left(\vec{v}_{\chi}\right)=\frac{n_{\chi}}{(2 \pi)^{3 / 2} \bar{v}_{\chi}^{3}} \exp \left[-\frac{\left(\vec{v}_{\chi}-V \hat{x}\right)^{2}}{2 \bar{v}_{\chi}^{2}}\right],
$$

is the dark-matter phase-space distribution, with $\bar{v}_{\chi}=$ $\left(k T / m_{\chi}\right)^{1 / 2}$. Recall also that we expect $V \ll \bar{v}_{p}$, as discussed above.

The drag-force per unit mass is obtained by integrating the momentum transfer per collision over all collisions between protons and dark-matter particles. From the symmetry of the problem, the deceleration of the darkmatter fluid will be in the $\hat{x}$ direction, and it will have a magnitude,

$$
\begin{aligned}
a_{x}= & \frac{1}{n_{\chi}} \int d^{3} v_{\chi} f_{\chi}\left(\vec{v}_{\chi}\right) \int d^{3} v_{p} f_{p}\left(\vec{v}_{p}\right)\left|\vec{v}_{p}-\vec{v}_{\chi}\right| \\
& \times \int d \Omega \frac{d \sigma}{d \Omega}\left(v_{\chi x f}-v_{\chi x i}\right) .
\end{aligned}
$$

Here $\Omega=(\theta, \phi)$ is the scattering angle in the center-ofmass frame, and $v_{\chi x f}-v_{\chi x i}$ is the difference between the final and initial $x$ component of the dark-matter-particle velocity; the difference is the same in the center-of-mass and laboratory frames. The differential cross-section $d \sigma / d \Omega$ is that given in Eq. (5).

Consider an individual scattering event. Let $\alpha$ be the angle that $\vec{v}_{p}-\vec{v}_{\chi}$ makes with the $\hat{x}$ direction; this is then the angle that $\vec{v}_{\chi}$ makes with the $\hat{x}$ axis in the centerof-mass frame, and the magnitude of the initial and final dark-matter velocities in the center-of-mass frame is $v_{\chi}^{\mathrm{cm}}=m_{p} v /\left(m_{p}+m_{\chi}\right)$, where $v \equiv\left|\vec{v}_{p}-\vec{v}_{\chi}\right|$ is the relative velocity. The initial $\hat{x}$ component of the dark-matter velocity in the center-of-mass frame is then $v_{\chi x i}=$ $v_{\chi}^{\mathrm{cm}} \cos \alpha$. The scattering angles $\theta$ and $\phi$ are then the polar and azimuthal angles that the scattered dark-matter velocity makes with the initial velocity in the center-ofmass frame. By rotating this coordinate system by an angle $\alpha$ about the $\hat{z}$ axis to align it with the laboratory $\hat{x}$ axis, we find $v_{\chi x f}=v_{\chi}^{\mathrm{cm}}(\cos \alpha \cos \theta-\sin \alpha \sin \theta \sin \phi)$. Thus,

$$
\int d \Omega \frac{d \sigma}{d \Omega}\left(v_{\chi x f}-v_{\chi x i}\right)=-\frac{m_{p} Z^{2} e^{2}\left(\mathcal{D}^{2}+\mathcal{M}^{2}\right) \cos \alpha}{2 \pi\left(m_{p}+m_{\chi}\right) v} .
$$

Completing the integral in Eq. (A3) in the limit $V \ll$ $\left(\bar{v}_{p}, \bar{v}_{\chi}\right)$, we find

$$
a_{x}=\frac{2(Z e)^{2}\left(\mathcal{D}^{2}+\mathcal{M}^{2}\right) m_{p} n_{p}}{3 \pi(2 \pi)^{1 / 2}\left(m_{p}+m_{\chi}\right)} \frac{V}{\sqrt{\overline{\boldsymbol{v}}_{p}^{2}+\overline{\boldsymbol{v}}_{\chi}^{2}}} .
$$

Taking into account the definition $\left\langle v_{p}\right\rangle=\sqrt{8 T_{p} /\left(\pi m_{p}\right)}$, this drag-force leads to the drag-force term in Eqs. (17), (20), and (21) when including the simple corrections for a mass fraction $Y$ of helium.
[1] F. Zwicky, Helv. Phys. Acta 6, 110 (1933).

[2] S. Tremaine and J. E. Gunn, Phys. Rev. Lett. 42, 407 (1979).

[3] See, e.g., http://pdg.lbl.gov.

[4] G. Jungman, M. Kamionkowski, and K. Griest, Phys. Rep. 267, 195 (1996).

[5] M. S. Turner, Phys. Rep. 197, 67 (1990); G. Raffelt, Phys. Rep. 198, 1 (1990); K. van Bibber and L. Rosenberg, Phys. Rep. 325, 1 (2000).
[6] G. D. Starkman, A. Gould, R. Esmailzadeh, and S. Dimopoulos, Phys. Rev. D 41, 3594 (1990).

[7] E. D. Carlson, M. E. Machacek, and L. J. Hall, Astrophys. J. 398, 43 (1992).

[8] D. N. Spergel and P. J. Steinhardt, Phys. Rev. Lett. 84, 3760 (2000).

[9] A. Gould, B. T. Draine, R.W. Romani, and S. Nussinov, Phys. Lett. B 238, 337 (1990). 
[10] See, e.g., S. Davidson, S. Hannestad, and G. Raffelt, J. High Energy Phys. 05, (2000) 003.

[11] S. L. Dubovsky, D. S. Gorbunov, and G. I. Rubtsov, JETP Lett. 79, 1 (2004); S. L. Dubovsky, D. S. Gorbunov, and G. I. Rubtsov, Pis'ma Zh. Eksp. Teor. Fiz. 79, 3 (2004).

[12] E. Fermi and E. Teller, Phys. Rev. 72, 399 (1947).

[13] S. Burles et al., Phys. Rev. Lett. 82, 4176 (1999).

[14] E.W. Kolb and M.S. Turner, The Early Universe (Addison-Wesley, Reading, MA, 1990).

[15] P. de Bernardis. et al., Nature (London) 404, 955 (2000); S. Hanany et al., Astrophys. J. Lett. 545, 5 (2000); N.W. Halverson et al., Astrophys. J. 568, 38 (2002); B. S. Mason et al., Astrophys. J. 591, 540 (2003); A. Benoit et al., Astron. Astrophys. L 399, 25 (2003); D. N. Spergel et al., Astrophys. J. Suppl. Ser. 148, 175 (2003).

[16] K. Griest and M. Kamionkowski, Phys. Rev. Lett. 64, 615 (1990).

[17] M. Kamionkowski and M. S. Turner, Phys. Rev. D 42, 3310 (1990).

[18] L. L. Foldy, Phys. Rev. 83, 688 (1951).

[19] CDMS Collaboration, D.S. Akerib et al., astro-ph/ 0405033.

[20] CDMS Collaboration, D. S. Akerib et al., Phys. Rev. D 68, 082002 (2003).

[21] G. Angloher et al., Astropart. Phys. 18, 43 (2002).

[22] P. C. McGuire and P. J. Steinhardt, astro-ph/0105567.

[23] J. Rich, R. Rocchia, and M. Spiro, Phys. Lett. B 194, 173 (1987).

[24] D. McCammon et al., Astrophys. J. 576, 188 (2002).

[25] Muon g-2 Collaboration, G.W. Bennett et al., Phys. Rev. Lett. 89, 101804 (2002); Phys. Rev. Lett. 89, 129903(E) (2002).

[26] Particle Data Group, K. Hagiwara et al., Phys. Rev. D 66, 010001 (2002).

[27] C. Bird, P. Jackson, R. Kowalewski, and M. Pospelov, hep-ph/0401195.

[28] BABAR Collaboration,B. Aubert et al., hep-ex/0304020.

[29] CLEO Collaboration, T.E. Browder et al., Phys. Rev. Lett. 86, 2950 (2001).

[30] BELLE CollaborationK. Abe et al., Phys. Rev. Lett. 88, 021801 (2002).
[31] BABAR Collaboration, B. Aubert et al., hep-ex/0107026.

[32] L3 Collaboration, P. Achard et al., Phys. Lett. B 587, 16 (2004); CDF Collaboration, D. Acosta, Phys. Rev. Lett. 92, 121802 (2004); CDF Collaboration, T. Affolder et al., Phys. Rev. Lett. 88, 041801 (2002).

[33] S. Davidson, B. Campbell, and D. Bailey, Phys. Rev. D 43, 2314 (1991).

[34] A. A. Prinz et al., Phys. Rev. Lett. 81, 1175 (1998).

[35] C. P. Ma and E. Bertschinger, Astrophys. J. 455, 7 (1995).

[36] Scott Dodelson, Modern Cosmology (Academic, New York, 2003).

[37] X. L. Chen, S. Hannestad, and R. J. Scherrer, Phys. Rev. D 65, 123515 (2002).

[38] K. Sigurdson and M. Kamionkowski, Phys. Rev. Lett. 92, 171302 (2004).

[39] F. E. Low, Phys. Rev. 96, 1428 (1954).

[40] M. Gell-Mann and M. L. Goldberger, Phys. Rev. 96, 1433 (1954).

[41] P. J. E. Peebles and J. T. Yu, Astrophys. J. 162, 815 (1970).

[42] M. Doran and C. M. Mueller, astro-ph/0311311.

[43] SDSS Collaboration, M. Tegmark et al., Astrophys. J. 606, 702 (2004).

[44] G. Hinshaw et al., Astrophys. J. Suppl. Ser. 148, 135 (2003).

[45] A. C. S. Readhead et al., Astrophys. J. 609, 498 (2004).

[46] C. Dickinson et al., astro-ph/0402498.

[47] A. G. Riess et al., Astrophys. J. 607, 665 (2004); J. L. Tonry et al., Astrophys. J. 594, 1 (2003); R. A. Knop et al., Astrophys. J. 598, 102 (2003); B. J. Barris et al., Astrophys. J. 602, 571 (2004).

[48] L. Bergstrom, P. Ullio, and J. Buckley, Astropart. Phys. 9, 137 (1998).

[49] M. Pospelov and T. ter Veldhuis, Phys. Lett. B 480, 181 (2000).

[50] M. Kamionkowski et al., Phys. Rev. Lett. 74, 5174 (1995).

[51] G. Raffelt, Stars as Laboratories for Fundamental Physics (University of Chicago, Chicago, 1996).

[52] J. Bagnasco, M. Dine, and S. Thomas, Phys. Lett. B 320, 99 (1994). 